\title{
Inconsistency in dairy calves' responses to tests of fearfulness
}

\section{Article}

\section{Accepted Version}

Creative Commons: Attribution-Noncommercial-No Derivative Works 4.0

Meagher, R. K., von Keyserlingk, M. A.G., Atkinson, D. and Weary, D. M. (2016) Inconsistency in dairy calves' responses to tests of fearfulness. Applied Animal Behaviour Science, 185. pp. 15-22. ISSN 0168-1591 doi:

https://doi.org/10.1016/j.applanim.2016.10.007 Available at https://centaur.reading.ac.uk/68219/

It is advisable to refer to the publisher's version if you intend to cite from the work. See Guidance on citing.

To link to this article DOI: http://dx.doi.org/10.1016/j.applanim.2016.10.007

Publisher: Elsevier

All outputs in CentAUR are protected by Intellectual Property Rights law, including copyright law. Copyright and IPR is retained by the creators or other copyright holders. Terms and conditions for use of this material are defined in the End User Agreement.

\section{www.reading.ac.uk/centaur}

\section{CentAUR}

Central Archive at the University of Reading 
Reading's research outputs online 
1 Inconsistency in dairy calves' responses to tests of fearfulness

2

3 Rebecca K. Meagher, Marina A.G. von Keyserlingk, Dax Atkinson, Daniel M. Weary

4

5 Animal Welfare Program, University of British Columbia, 2357 Main Mall, Vancouver, BC,

6 Canada V6T $1 Z 4$

7

8 Corresponding author: Rebecca K. Meagher, Email: rkmeagher@gmail.com

9

10 


\section{Abstract}

Fear is an important welfare problem for farm animals including cattle. A variety of

14 methods of assessing fear have been proposed, but the reliability and validity of these methods,

15 and ways of improving these characteristics, have received little study. We conducted a series

16 of experiments to assess the consistency of dairy calves' responses of novel objects and to

17 humans, and to investigate factors that might improve reliability. In the first experiment, latency

18 to touch a novel object had moderate reliability $\left(r_{s}=0.54\right)$, and latency to touch a stationary,

19 familiar human had negligible reliability $\left(r_{s}=0.26\right)$. Experiment $2 a$ used the same test protocols,

20 but with a shorter interval between repeat testing and using different stimuli in the two novel

21 object tests; this change did not improve reliability (e.g. $r_{s}=0.29$ for the novel-object test).

22 Reliability for this test was improved $\left(r_{s}=0.58\right)$ in Experiment $2 b$, when the same object was

23 used in both tests rather than a truly novel object being used the second time. Experiment $2 a$

24 found ceiling effects in the response to human test associated with the short period during which

25 approach responses were recorded. High reliability was found in Experiment 2b, where the

26 maximum test duration was doubled, but this effect not due to the extended duration.

27 Experiment 3 assessed reliability of a response to human approach at the farm rather than

28 individual level, in this case assessing responses to an unfamiliar person. The proportion of

29 calves making contact with the person was not reliable $\left(r_{s}=0.22\right)$, but the proportion retreating

30 from the person had moderate reliability $\left(r_{s}=0.52\right)$. Reliability was improved by excluding data

31 from calves that had coughs on the day of testing. Conducting multiple tests per individual using

32 different stimuli and reporting health status of the animals are recommended for future research

33 and animal welfare assessment schemes that include measures of fear.

\section{Keywords}


37 Fearfulness; neophobia; human-animal relationship; well-being; reliability; validity

\section{Introduction}

Fear is widely recognized as a welfare concerns for cattle and other farm animals (e.g.

42 Farm Animal Welfare Council 2009; Hemsworth et al. 2000; Jones and Boissy 2011). Fearful

43 animals can also cause production and management challenges, including decreased

44 productivity (e.g. Barnett et al. 1992; Hemsworth et al. 2000) and animals that are afraid of

45 humans may be more dangerous to handle (Boivin et al. 1992; Hemsworth et al. 1989).

46 Unfortunately, methods of assessing fear (a negative emotional state resulting from a perceived

47 threat [Gray 1987; Ennaceur 2014]) and fearfulness (a personality trait characterized by a

48 tendency to express fear when exposed to potentially threatening stimuli or situations) appear

49 not to be well-validated and have uncertain reliability (Forkman et al. 2007). Of 112 papers

50 published in this journal over a five-year period ending in August 2015 with fear* or anx* in the

51 keywords, abstract or title, only 65 papers (or $58 \%$ ) contained any form of the words reliable or

52 repeatable anywhere in the text, and of these, only 15 actually estimated reliability. Measures

53 also vary considerably across studies, making it difficult to extrapolate results from one

54 approach to the next (Forkman et al. 2007).

55 The need for valid, reliable ways of assessing welfare in farm animals is widely

56 recognized, to be used for example in assurance schemes for commercial farms (see Scott et

57 al. 2001). Currently, fear is often assessed in farm animals through response to novelty

58 (neophobia, although other factors such as exploratory motivation also influence the response),

59 most commonly using a novel object test. Another common type of fear-related test is in

60 response to humans (e.g. Forkman and Keeling 2009), as fear of handlers may have a major

61 impact on the lives of intensively farmed animals. Research published to date indicates that 
62 responses are not closely associated in these two contexts (e.g. Hegelund and Sorensen 2007),

63 and that separate measures may be needed. From the perspective of animal welfare,

64 fearfulness and long-lasting states of fear are of special interest, meaning that we are especially

65 interested in fear responses that are consistent over time. Unfortunately, test-retest reliability

66 (also called repeatability) is often weak making it difficult to draw strong inferences from a single

67 test.

In cattle, for example, the novel object test was reported to be reliable within individuals

69 between tests in at least two calf studies (using measures derived from factor analysis in Van

70 Reenen et al. 2004, and approach latency in Bokkers et al. 2009), but was unreliable in older

71 heifers and adult cows when tested using avoidance (Van Reenen et al. 2013), reactivity

72 (Gibbons et al. 2009), number of interactions and time in proximity (Kilgour et al. 2006). Results

73 have been mixed across a range of measures and ages in other studies (Graunke et al. 2013;

74 MacKay et al. 2014). Even the methods of assessing 'repeatability' vary: while most studies

75 replicate the test exactly using the same stimulus, others (e.g. Gibbons et al. 2009) instead assess consistency of response across different novel stimuli because there is no way to repeat

77 a test and have it be truly novel (see e.g. Forkman et al. 2007 for a discussion of this problem).

78 Nonetheless, the novel object test has face validity, meaning that it appears sensible based on

79 our understanding of fear and comparisons with human behaviour, as judged by experts (e.g.

80 Scott et al. 2001; Whay et al. 2003). It is also one of the few tests that has undergone some

81 successful validation for cattle, suggesting it may be a true indicator of fear (based on

82 correlation with other fear- and stress-related measures and pharmacological validation using

83 anxiolytic drugs; e.g. Van Reenen et al. 2005; Van Reenen et al. 2009). Confirming or finding

84 ways to improve its reliability would thus be valuable.

85 Responses to humans (typically measured as approach or avoidance by the animal) are

86 more consistently reported to be reliable (at the individual level in calves [Rousing et al. 2005]

87 and cows [Gibbons et al. 2009; Turner et al. 2011]). However, some papers found moderate to 
88 high repeatability only for some measures and time periods (Haskell et al. 2012; Mazurek et al.

89 2011; Windschnurer et al. 2008; Windschnurer et al. 2009; see also review of responses to

90 humans by de Passillé and Rushen 2005), and other studies have found no repeatability (Battini

91 et al. 2011), although all of these studies depended on some measure of avoidance or retreat

92 from a human. Fina and colleagues (2006) reported that reliability of responses to restraint

93 differed depending upon the calves' initial responses, with calm individuals remaining calm

94 across tests but fearful ones showing reduced fear over time.

95 Farm-level repeatability is also important for measures of approach or avoidance of

96 humans, because this type of measure has been proposed for use in on-farm welfare

97 assessments (e.g. Winckler et al. 2003; Winckler et al. 2007), focussing on herd-level

98 differences. Only a few papers have investigated farm-level repeatability of responses to

99 humans, all in adult cows, and studies have sometimes confounded test-retest reliability with

100 inter-observer reliability (e.g. Windschnurer et al. 2009). In these tests (based upon avoidance

101 of an approaching human) low to moderate reliability has been reported (De Rosa et al. 2003;

102 Winckler et al. 2007). Reliability can also be estimated at the level of the pen or group

103 (intermediate between individual and farm levels), and indeed some farm level estimates are

104 based upon observations of a single pen. Only one study on calves has assessed the reliability

105 of approach responses measured at the pen level, and this study reported high reliability

106 (Bokkers et al. 2009, with similar results for an avoidance measure).

107 Even among papers that claim repeatability, correlations are sometimes low. For

108 example, Turner and colleagues (2011) assessed repeatability across and within tests of fear of

109 humans in beef cattle and found the proportion of variance explained by individual consistency

110 ranged from 0.17 to 0.54 . In fact, a meta-analysis of the personality literature in wild animals

111 found an average repeatability (intraclass correlation coefficient) of only 0.37 (Bell 2009), which

112 is considerably below the level generally deemed acceptable ( 0.6 being a traditional standard in

113 the human literature (e.g. Bruton et al. 2000, Mroczek 2007). In humans, typical correlations 
114 over long intervals (years) are often over 0.7 in adults (Mroczek 2007). Conversely, correlation

115 coefficients for children and college students were only 0.31 and 0.54 respectively, for major

116 personality traits in one meta-analysis (Roberts and DelVecchio 2000). It therefore seems likely

117 that other juvenile animals, such as calves, may also show limited correlations in their fear

118 responses over time.

119 The aims of the current study were to assess the individual-level test-retest reliability of

120 versions of novel object and response to human tests, and the farm-level test-retest reliability of

121 a response to human test. An additional aim was to identify factors that influence reliability,

122 enabling refinements in protocols used in future research and on-farm welfare assessments.

123 The factors investigated included consistency of the object used in the novel object test, test

124 duration, and calf health. We also assessed inter- and intra-observer reliability (i.e. consistency

125 between and within people recording the data) of the measures, as these are essential to 126 obtaining test-retest reliability.

\section{Materials and methods}

\subsection{Experiment 1}

All of the research presented in this paper was approved by the University of British

133 Columbia Animal Care Committee. In this experiment we used 32 Holstein bull calves, housed

134 at the University of British Columbia Dairy Education and Research Centre. These calves also

135 served in a concurrent study on the effects of early social housing, comparing individually

136 housed calves $(n=10)$, pair-housed calves $(n=12)$, and calves kept in a complex social group

137 with access to their dams $(n=10)$. More detail regarding these treatments is available in

138 Meagher et al. (2015). Pens were cleaned once per week. Calves were offered $8 \mathrm{~L}$ of milk per

139 day for the first $28 \mathrm{~d}$, at which time the milk ration was reduced to $6 \mathrm{~L}$ over $3 \mathrm{~d}$, always split 
between two daily feedings. This reduction was intended to stimulate solid feed intake. At

141 approximately $58 \mathrm{~d}$, calves were weaned over a 3-day period. Calves had ad libitum access to 142 water throughout the experimental period, and access to grain (Hi-Pro Medicated Calf Starter)

143 and a mixed ration beginning at day $5 \pm 2$. Health checks were performed weekly throughout the

144 experimental period to assess symptoms of common illnesses, including respiratory and enteric

145 disease. Calves were treated when appropriate according to standard farm protocols.

146 Two tests for fearfulness were used: novel object and response to human (in this case

147 approach to a stationary, familiar person). These tests were conducted on consecutive days at

148 approximately $41 \mathrm{~d}$ of age and repeated at approximately $62 \mathrm{~d}$ of age. The response to human

149 test was also conducted at $25 \mathrm{~d}$ of age. Tests were conducted between the two daily feedings,

150 but never within 30 min of either feeding time. Novel object tests took place in a test pen that the

151 calves had visited twice daily (for cognitive training; see Meagher et al. 2015) for several weeks.

152 After 2 min of habituation to the pen, the novel object (in this case, a brightly coloured ball) was

153 lowered into the pen using a length of twine. The test lasted $10 \mathrm{~min}$, and latency to make

154 contact with the ball was recorded. The response to human tests were conducted during weekly

155 weighing of the animals, following a similar procedure to Duve and colleagues (2012) in which

156 calves were allowed to approach a human and then their response to weighing was assessed.

157 In brief, the calf was released from its pen into the alley, and given up to $90 \mathrm{~s}$ to make contact

158 with the stationary person. The stationary person (one person per experiment) was familiar to

159 the calves and stood $2.4 \mathrm{~m}$ away. The first author (RKM, who was also familiar to the calf) stood

160 inside the pen and recorded the latencies to touch the person. Wooden dividers blocked the

161 view of calves on the other side of the aisle, leaving an alley approximately $1.2 \mathrm{~m}$ wide for the

162 individual and pair treatments; however, calves could see into neighbouring pens on the same

163 side of the alley as they approached the person. For the group-housed calves, the distance to

164 the person was equivalent, but the space was wider and no other calves were in sight. The calf

165 was then encouraged or pushed onto the scale (by the previously stationary person), and the 
166 difficulty of pushing was scored by the handler on a scale of 0 to 4 , with 0 indicating the calf 167 walked onto the scale with no physical guidance, and 4 that a single handler could not get them 168 on the scale alone.

169 Test-retest reliability was assessed using Spearman rank correlations due to non170 normality of the data. Weighted sums of Spearman correlations are presented to control for 171 effects of housing treatment (Taylor 1987). Correlation coefficients and not $p$-values are 172 reported, because $p$-values are too dependent on sample size to be very useful measures of 173 reliability (Martin and Bateson 2007). Throughout the paper, we categorize reliability as 174 negligible (correlation <0.30), low (0.30-0.49), moderate $(0.50-0.69)$, high $(0.70-0.89)$ or very 175 high $(\geq 0.90)$ following Hinkle and colleagues (2003). For the ordinal data from scores of difficulty 176 of handling during weighing, we used two types of analysis: kappa scores for agreement on the

177 ordinal data (categorized according to Dohoo et al. 2002), and kappas combined with percent 178 agreement when converted to a binary analysis for some force needed (scores 2 to 4 ) versus no 179 force needed (scores 0 or 1 ).

\subsection{Experiment 2}

\subsubsection{General methods}

In Experiment 1, the testing schedule was partially determined by the other experiment

186 running simultaneously, and the calves had some experiences between tests that might have 187 caused changes in behaviour, including weaning from milk onto solid feed. Thus, in Experiment

1882 we assessed the reliability of the handling and novel object responses using a shorter inter189 test interval and during a period of consistent management.

$190 \quad$ The subjects were two cohorts of Holstein calves. In Experiment 2a we used 27 calves 191 (18 male, 9 female), and in Experiment 2b we used 13 calves (all female). Calves were 
192 individually housed until the end of the experiment and cared for in the same way as described

193 above, except for the following differences in feeding: no total mixed ration was provided during

194 this experiment, and calves were stepped down to $4 \mathrm{~L}$ of milk rather than to $6 \mathrm{~L}$ beginning at $d$

195 26. Also, for the purposes of a related experiment, 13 of the calves in the first group were given

196 a nutritional supplement with their milk, alpha S1 casein hydrosylate (Zylkène ${ }$, distributed by

197 Vétoquinol, Princeville, QC), beginning $7 \mathrm{~d}$ before the start of fear testing and ending on the day

198 they were moved to the group pens. This treatment did not affect any of the response measures

199 except latency in the first novel object test.

\subsubsection{Experiment $2 a$}

202

At age $36 \pm 3 d$, a novel object test was conducted in the home pen. This test was

204 repeated $7 \mathrm{~d}$ later $(\mathrm{d} 43 \pm 3$ ). Two different objects were used to maintain the novelty of the test

205 rather than conducting an exact replicate: a red and white ball, and a blue plastic basket. Half of

206 the calves received the ball in the first test and the basket in the second, and the other half

207 received the objects in the reverse order. The tests were conducted in the same way as

208 Experiment 1, but in addition to latency to make contact, total time in contact with the object was

209 recorded. All measures were assessed from video recordings by trained observers (one per

210 variable) who were blind to the study aims, and intra-observer reliability was tested by having

211 these observers score a subset of the videos a second time to ensure that they were consistent

212 in their scoring; latency to make contact was also recorded live for all calves by the first author,

213 who also assessed the other measures from a subset of videos for inter-observer reliability

214 testing. Responses to a human handler were also assessed as in Experiment 1. These tests

215 were conducted on the day following each novel object test.

$216 \quad$ Normality of the data was assessed using Shapiro-Wilk tests. Latency data were non-

217 normally distributed, so repeatability was assessed using Spearman rank correlations. Difficulty 
218 of handling scores were analysed as in Experiment 1. Six calves showed symptoms of illness at

219 some time during the testing period, primarily with enteric illness, which may have affected

220 reaction speed and likelihood of approaching the object; these calves were excluded from the 221 analyses.

\section{2.2.3. Experiment $2 b$}

The second cohort of calves was used to assess whether modifying the protocols used

226 in Experiment 2a would improve reliability. Housing, care and testing protocols were the same

227 as in Experiment 2a, except for one change in each test. For the novel object tests, the same

228 object was used in both tests (each calf being assigned to either the ball or the basket) rather

229 than calves getting a different object in Test 1 and 2. For the response to human tests, the

230 duration of the test was extended from $90 \mathrm{~s}$ to $180 \mathrm{~s}$ to reduce potential ceiling effects. The data

231 were analysed for test-retest reliability as above, again excluding calves that were ill.

\section{2.3. Experiment 3}

This experiment was conducted on 15 dairy farms in the Fraser Valley of British

236 Columbia, Canada, with the aim of assessing farm-level repeatability in response to humans.

237 Unweaned calves between 7 and $70 \mathrm{~d}$ of age were tested. Because each farm was visited

238 twice, 6 to 8 wk apart, the individual calves tested on the second visit were a completely

239 separate cohort, but represented the full range of ages where possible (average age in test 1:

24034 ; test 2: 37 d). All calves were Holstein or Holstein crosses. Data were collected from a total

241 of 677 calves, with an average of 21 calves per farm on each visit. Tests were conducted

242 between morning and afternoon feedings and never within an hour of feeding time. 
Fear of humans was assessed using an approaching human test, which could be

244 conducted without opening the calf pens. Unlike in the previous experiments, the human (RKM)

245 was unfamiliar to the calves. The person walked along the row of pens, parallel to them and

246 approximately $1 \mathrm{~m}$ from the front of each pen or hutch (space permitting). Once directly in front

247 of a pen, she then turned to face the calf and said "hello" to attract their attention (cf. Bokkers et

248 al. 2009). After pausing for $5 \mathrm{~s}$ to record any locomotor response, she approached the calf at a

249 pace of approximately 1 step per second (as in e.g. Windschnurer et al. 2008), and then

250 extended her arm to where the calf could reach it, with the hand flat and oriented sideways.

251 Direct eye contact was avoided (Bokkers et al. 2009). Retreats were scored on an ordinal scale

252 according to Table 1 . We also recorded whether the calf touched the experimenter, and the

253 latency to do so, within $2 \mathrm{~min}$. The experimenter then repeated the procedure at the next pen in

254 the row, following the same route through the pens on both visits to a farm, and never passing

255 directly in front of a calf prior to its test if at all possible. For socially-housed calves, latencies

256 and retreats for each calf in the pen were recorded.

257 Calf health was visually assessed after each test. The presence of a spontaneous

258 cough, or faecal consistency scoring greater than 2 (following McGuirk, 2013) were considered

259 indicators of illness.

260 Repeatability of the test was assessed at the farm level for the proportion of calves

261 making contact with the experimenter, since calves within a farm were non-independent, using a

262 Spearman rank correlation. Repeatability of retreats in this test was also assessed with

263 Spearman rank correlations, using three different ways of summarizing the behaviour:

264 proportion of calves retreating by the time the experimenter was at the pen with hand extended

265 (score 2 or above) or prior to extending the hand (score 3 or above), and the average score for 266 each farm.

267 One farm was excluded because a major housing change occurred between tests. On

268 the remaining 14 farms, individual calves were excluded if they showed signs of diarrhoea or 
269 respiratory illness or both, based on the criteria above. The reliability analyses were then

270 repeated to check for an effect of these illnesses on the results.

271

\section{3. Results}

273

\section{3.1. Experiment 1}

275

Latencies to approach the novel object were moderately correlated between tests at 42

277 and $60 \mathrm{~d}$ of age, with a correlation coefficient $\left(r_{s}\right)$ of $0.54(n=24$; Figure 1). There was little

278 evidence of any relationship in approach latencies to the human handler between tests at 25

279 and $42 \mathrm{~d}$ of age $\left(r_{s}=0.26, n=23\right)$, nor at 42 versus $60 \mathrm{~d}$ of age $\left(r_{s}=0.21, n=26\right)$.

280 Difficulty of handling scores showed low reliability using the ordinal scale. Kappa values

281 were 0.33 for day 25 vs. 42 and 0.22 for day 42 vs. 60 (indicating "fair agreement": Dohoo et al.

282 2003). However, 22 of 31 calves (71\%) were consistent from days 25 to 42 in terms of whether

283 any force was needed (kappa 0.44 , indicating moderate reliability). For day 42 vs. 60 , percent

284 agreement was similar: 23 of 34 calves (68\%; kappa 0.35$)$.

\subsection{Experiment 2a}

\subsubsection{Test-retest reliability}

The correlation between Tests 1 and 2 for latency to touch the novel object was

291 negligible $\left(r_{s}=0.29, n=20\right.$; Figure 2a). Excluding calves that failed to touch the object in at least

292 one test, which often happened if calves were resting immediately before the test, perhaps

293 reflecting drowsiness rather than increased fear or lack of interest, improved the correlation

294 between tests $\left(r_{s}=0.70, n=15\right)$. The reliability of time in contact with the object was low when 
295 considering all calves $\left(r_{s}=0.30, n=20\right)$, and negligible when excluding those that did not make 296 contact $\left(r_{s}=0.02, n=16\right)$.

297 For the response to humans, a correlation between latencies in the two tests could not

298 be meaningfully assessed because only 6 of 27 calves ever made contact with the handler on

299 the first test, and of these only three also made contact during the second test. Agreement in

300 difficulty of handling scores was very low whether data were analysed as ordinal or binary

301 (kappa 0.07 and 0.03 , respectively), although there was 50\% agreement in the latter (10 of 20

302 calves).

303

304 3.2.2. Intra- and inter-observer reliability

305

Inter-observer reliability for latency to touch the novel object was very high $\left(r_{s}=0.93\right.$,

$307 \mathrm{n}=27$ ), and intra-observer reliability was also high for the subset of videos that were re-assessed

$308\left(r_{s}=0.81, n=15\right)$. Total time in contact also had high inter-observer reliability $\left(r_{s}=0.70, n=10\right)$ and

309 very high intra-observer reliability $\left(r_{s}=0.94, n=15\right)$.

311 3.2.3. Experiment $2 b$

312

313 Test-retest reliability for latency to approach the novel object was higher in this

314 Experiment $\left(r_{s}=0.58, n=11\right.$; Figure $\left.2 b\right)$, but excluding non-contacts did not improve reliability

$315\left(r_{s}=0.32, n=10\right)$. Reliability of the response to human was high in this experiment, $\left(r_{s}=0.76\right.$,

$316 \mathrm{n}=10$; Figure 3). However, this improvement was not the result of using the extended maximum

317 test duration of $180 \mathrm{~s}$; only 1 calf made contact with the handler between 90 and $180 \mathrm{~s}$ on both

318 tests, and artificially imposing a $90 \mathrm{~s}$ ceiling produced a high reliability coefficient $\left(r_{\mathrm{s}}=0.83\right.$,

$319 \mathrm{n}=10)$. The high reliability was partially due to the fact that failure to make contact within $90 \mathrm{~s}$

320 was consistent among individuals: 5 of the 6 who did not make contact on the first test also 
321 failed to make contact in the second test. Agreement in difficulty of handling scores was fair for 322 this group (kappa 0.26), and this value was similar $(0.27)$ for whether any force was needed to 323 get the calf on the scale, with 7 of $11(64 \%)$ calves in agreement.

\subsection{Experiment 3} presented in Table 2. In brief, the proportion of calves making contact with the person showed

329 low or negligible repeatability; indeed, the slope of the relationship was negative. Retreats were

330 moderately repeatable for the full data set. Using yes/no data for whether a calf retreated at all,

331 before the person's arm was extended (score 3 or above) was slightly more reliable than

332 including retreats at the time the arm was extended (score 2). The most reliable measure was 333 the average retreat score for the farm.

334 Signs of illness were recorded for 68 of 599 calves on the 14 farms analysed. For three

335 of the four response variables, excluding calves with coughs improved repeatability. Excluding 336 calves with diarrhoea only improved repeatability for two response measures, and excluding 337 both groups reduced repeatability for all measures relative to excluding coughs alone.

\section{4. Discussion}

\subsection{Factors influencing repeatability}

The results show varying levels of repeatability in both novel object tests and those

344 assessing response to humans. We speculated that the low reliability in Experiment 1 was due

345 to a long test-retest interval (approx. $20 \mathrm{~d}$ ), combined with important management changes

346 (including weaning from milk). Consistent with this idea, we found some improvement in 
347 reliability estimates for the novel object test in some groups when we switched to shorter 348 intervals (7 d) with more consistent management (pre-weaning only) in Experiment 2, and for 349 the response to human test in Experiment 2b. Agreement in scores of difficulty of handling was 350 typically low to fair across the experiments, although it was higher for the binary (some force vs. 351 no force needed) scale than the ordinal scale in Experiment 1.

352 In Experiment 2a, the improvement in novel object reliability occurred only when

353 including animals that were alert during testing. Unfortunately, the results of this inclusion

354 criteria differed between Experiments $2 a$ and b, which may reflect some instability in the 355 correlation estimates due to the small sample sizes available (see e.g. Goodwin and Leach 356 2006). Based on the human literature, the sample sizes needed for stability of personality 357 correlation estimates would be very difficult to achieve (e.g. n=250: Schönbrodt and Perugini 358 2013); we suggest instead the use of multi-study replication, ideally with meta-analyses, to 359 confirm the reported effects. However, the result from 2a suggests that it would be worthwhile to 360 investigate the benefit of a further refinement that could be used for both the novel object test 361 and human approach tests conducted in the home pen: imposing a procedure or criteria to 362 ensure that animals were attending to the test situation. For example, in Experiment 1 calves 363 were moved to a testing pen and the test began shortly afterwards. This ensured that no calves 364 were asleep or resting at the time the stimulus was presented, as well as removing possible 365 distractions such as the presence of food. Home pen tests are desirable for practical reasons 366 and because they avoid introducing handling effects and social isolation for group housed 367 animals (see Forkman et al. 2007; Tecott and Nestler 2004), but in this case it seems that the 368 costs may outweigh the benefits (the reverse may be true when measuring exploration rather 369 than fear; see Carter et al. 2013).

370 In Experiment 2, we considered two additional factors thought to improve repeatability:

371 increasing the test duration when latencies are measured, and the consistency of the novel 372 object. Repeatability of the latency to touch humans could not be assessed in Experiment 2a 
373 due in part to ceiling effects associated with a short test; we thus hypothesised that increasing

374 the time allowed would improve reliability for the latency measures. The latencies in Experiment

$3752 \mathrm{~b}$ did show high reliability, but this was not due to the longer tests. That said, given that ceiling

376 effects prevented discrimination among individuals in Experiment 2a, we still contend that longer

377 test durations improve the validity and usefulness of the test by avoiding an artificial upper limit

378 in measures of latency. Others have similarly argued that extending test durations improves test

379 validity (e.g. in tests of chronic anxiety in rodents; Fonio et al. 2012).

380 The improved repeatability of the novel object in Experiment $2 b$ versus 2a was likely due

381 to using a second presentation of the same object. In Experiment 2a we had used a different

382 novel object for each test (to retain the novelty), but a disadvantage of this approach is that

383 animals may find some objects inherently more fear-inducing than others thus making

384 responses more variable. Although we found that using the same 'novel' object for multiple tests

385 improved the repeatability of the test, we do not recommend this practice in future tests. Instead

386 we argue that there is much to be gained from examining a range of objects; if individual

387 rankings differ between arbitrarily chosen objects with no apparent biological significance, it is

388 likely not valid to draw broad conclusions regarding 'fear of novelty' from tests with a single

389 object.

390 Experiment 3 identified the role of sickness, particularly respiratory illness, in reducing

391 reliability of responses to humans. Sickness behaviour is widely accepted to include lethargy

392 and decreased exploratory behaviour (e.g. Millman 2007; Swiergiel and Dunn 2007). A recent

393 study in calves found that respiratory illness and fever decreased probability of calves

394 approaching novel objects and stationary humans; diarrhoea did not immediately have this

395 effect, although during recovery from this ailment calves were less likely to approach people

396 (Cramer and Stanton 2015). Changes in health status could thus reduce repeatability of the

397 results for both types of test. Cramer and Stanton (2015)'s findings mirror the current results, in

398 that excluding calves with signs of respiratory illness most consistently improved the correlation 
between tests across variables. Excluding calves with signs of diarrhoea or both illnesses was

400 less helpful, although this may have been due to the reduced sample size (Goodwin and Leech

401 2006), and this should therefore be retested in a larger sample of calves. While the differences

402 in reliability estimates in this experiment were relatively small, collectively, these findings

403 support our choice to exclude animals that were sick around the day of testing in Experiment 2.

404 Unfortunately, health checks were not conducted on test days in Experiment 1. In future, health

405 status should be addressed when reporting responses to these tests.

406 Another lesson from Experiment 3 was that the proportion of calves making contact with

407 an unfamiliar human has low repeatability relative to other response measures. This is

408 surprising since this measure, and the related measure of latency to contact, are commonly

409 used (e.g. Bokkers et al. 2009; Forkman and Keeling 2009). We found that the most reliable

410 response measure was the retreat score. For retreat as a yes/no variable, which is simpler to

411 record, particularly when calves are group-housed, the correlation between visits was slightly

412 higher when counting retreats before the researcher's arm was extended versus retreats at the

413 time the arm was extended. Although the difference was small, it may reflect inconsistency in

414 the behaviour of the test person, such as speed of arm extension or positioning of the hand

415 relative to the calf. Repeatability of the retreat measures at farm level was comparable to the

416 individual-level results using latencies in Experiment 1.

417 Several factors that could influence repeatability of tests of fear were not investigated

418 here. As described by Waiblinger and colleagues (2006), human-animal relationship tests in

419 farm animals can be influenced by many factors, including interference by neighbouring

420 animals, exploratory, social, feeding and lying motivations, and social isolation. Feeding

421 motivation was relatively constant within each of the experiments in the current study (as tests

422 were held outside of regular feeding times, although this was not a perfect control since the

423 testing window was relatively large for practical reasons, likely increasing variation between

424 days), and social motivation and responses to isolation were not relevant in most cases. 
425 Interference by neighbouring animals was not an issue during the novel object tests, since the 426 calves were alone during testing, and were minimal throughout Experiment 3 since most calves

427 were housed alone and vocalizations were not common. However, it may have been an issue in 428 the response to human tests of Experiments 1 and 2, as calves could walk past the pens of 429 neighbours. In Experiment 3, there may also have been fluctuations in farm practice such as 430 staff members providing most care to the calves, or feeding times. However, this will be the 431 reality for any on-farm work and such variation must be accepted except where changes are 432 predictable (e.g. due to season) and can thus be accounted for in the study design.

433 One effect that has not been directly investigated in this context, but which is known to 434 play a role in animals' responses to potentially threatening stimuli, is laterality. Vertebrates, 435 including cows, typically prefer to view threatening stimuli from the left eye (Robins and Phillips 436 2010), and the eye that first sees a stimulus can influence escape responses (e.g. Austin and

437 Rogers 2007). It would be of interest to test whether inconsistency in the orientation of cattle 438 relative to fear-inducing stimuli can explain differences in responses on repeated tests. Testing 439 this idea will require a test environment that allows control of presentation side.

Even if protocols are refined to reduce noise, there are likely limitations in the level of 444 repeatability that can be achieved. As discussed in the Introduction, the average repeatability 445 reported for personality traits of wild animals is only 0.37 (Bell et al. 2009). How consistency of 446 behaviour in farm animals will compare is difficult to predict. As de Passillé and Rushen (2005) 447 point out, even where there are moderate, statistically significant correlations, a large number of 448 animals will be "misclassified" by a single test. These limitations do not necessarily prevent the 449 tests from being useful; despite their typically low reliability, personality tests in wild animals can 450 still predict ecologically or practically important outcomes (e.g. Smith and Blumstein 2008). In 
451 the experiments described here, despite low to moderate reliability, the tests conducted at $41 \mathrm{~d}$

452 of age in Experiment 1 were able to detect some effects of treatment that correspond with

453 theory: fear of novelty was higher in calves reared in simpler, more socially restricted housing

454 (Meagher et al. 2015). Human personality studies typically report repeatability estimates

455 averaging 0.7 to 0.8 for the Big Five factors of personality (e.g. Gnambs 2014, Mroczek 2007),

456 but these factors are typically derived from multi-item scales. Having only one or two measures,

457 which is the norm in animal studies, is expected to increase measurement error (Credé et al.

458 2012).

459 A common recommendation when assessing traits is to conduct repeated tests and sum

460 or average responses. However, in the case of novelty, repeated testing is logically problematic

461 (see Forkman et al. 2007), as the object is no longer novel when presented a second time; even

462 if the object is changed, the test procedure becomes less novel. One approach to circumvent

463 this difficulty is to consider decreases in fear as an acceptable result when assessing reliability

464 (e.g. Meagher et al. 2011); repeated testing can then still be used to draw inferences, because

465 differences in habituation or sensitization rates may also be consistent, welfare-relevant

466 individual traits (Jones and Boissy 2011).

467 The results from the current study suggest that multiple tests might be needed, but using 468 a range of objects or other stimuli, given the differences in individual rankings depending on the 469 objects used. Similarly, Ramos (2008) argues that for measuring trait anxiety (and/or modelling 470 human anxiety disorders), conducting multiple types of tests is necessary. He argues that these 471 should be conducted simultaneously if the alternative is placing the animal in the same test 472 chamber or apparatus multiple times, but this would not allow assessment of how much of the

473 response is due to temporary states present at the time of testing. One difficulty with

474 recommending multiple tests is the time and expense, for example, of conducting multiple visits

475 to farms for on-farm welfare audits. Current protocols sometimes focus on ensuring inter- 
476 observer reliability (e.g. Wemelsfelder and Lawrence 2001), but our results indicate that this is

477 not sufficient for producing repeatability.

$478 \quad$ Farm-level repeatability could conceivably be attained without individual-level

479 repeatability, if problems with the latter are due only to the inherent problems in repeating a test

480 involving novelty. As long as the results are repeatable within farm using new groups of animals,

481 this would not be a major concern for farm-level investigations. Understanding why results

482 change within individuals is nonetheless important, since differences due to age or season

483 should be taken into account when selecting samples and testing times (see Haskell et al.

484 2012).

485

\subsection{Outstanding concerns regarding test validity}

The discussion above was focused primarily on practical issues regarding reliability of fear tests, but even if these issues can be resolved questions remain about test validity. Very

490 little validation testing has been done for response to human tests in calves, including the

491 voluntary approach-type tests used here, although some studies indicate that both voluntary

492 approach to humans and avoidance distance are influenced by rough or gentle handling

493 (Lensink et al. 2000; Schuetz et al. 2012; Windschnurer et al. 2009). The tests of neophobia

494 used in farm animals, such as novel object (e.g. Misslin and Ropartz 1981) and open field tests

495 (Hall 1936; Archer 1973), are largely adapted from tests originally developed and validated for

496 laboratory species. In some cases, the rationale for the test was based on the behavioural

497 ecology of the rodent species, and applicability to other species is questionable. For example,

498 the open field test makes sense for rats and mice that fear open areas (presumably because

499 these are associated with increased predation risk; e.g. Lister 1987; Ohl 2003; Rodgers 1997),

500 but cattle are too large to be at risk of overhead predators and are adapted to life in open

501 habitats. The novel object test is expected to apply more broadly (e.g. Russell 1973), but in 
some cases species-specific responses, such as burying, need to be taken into account (e.g.

503 Misslin and Ropartz 1981). As noted above, the object-specificity of the test results in

504 Experiment 2 also raises some concerns about its validity as a general measure of neophobia.

505 A second potential problem is that, even in laboratory animals where these tests have been

506 better validated and sometimes proved useful in drug screening, the validity of some tests (e.g.

507 the open field) has also been called into question (e.g. Ennaceur 2014). Known issues from the

508 laboratory animal literature include sensitivity to environmental variables unrelated to the

509 intended treatment, reducing external validity (Garner 2005) and preventing accurate measures

510 of trait anxiety because they are overshadowed by the effects of temporary states (e.g. Ohl 2003; Sylvers et al. 2011). This is likely one reason for failures to replicate results in different

512 laboratories (e.g. Dawson and Tricklebank 1995; Sousa et al. 2006; Wurbel, 2002).

513 Although careful attention to the methodological factors described above will likely

514 reduce problems of poor reliability and aid in the interpretation of data, the use of short-term

515 tests may be inherently problematic if the aim is to assess consistent traits in animals.

516 Temperament ratings by people who can integrate behaviour over time are one suggested

517 alternative (see Carlstead et al. 1999; Meagher 2009), but the relationship between these

518 measures and standard tests is not well understood (e.g. de Passillé and Rushen 2005). Finally,

519 the same underlying motivation can be expressed very differently depending on the testing

520 situation (e.g. approaching to bury an object when possible versus retreating from it if not),

521 potentially leading to misinterpretations regarding fearfulness (Franks et al. 2012). More

522 species-specific validations of the different types of fear test, taking into account natural

523 behaviour, are thus needed.

\section{5. Conclusions}


Moderate test-retest reliability seems achievable for both novel object and response to

528 human tests in dairy calves. It is, however, contingent on allowing sufficient time for the

529 behavioural response, and excluding calves with respiratory illness and perhaps other forms of

530 illness if replications of this work can confirm that they decrease reliability. In the case of novel

531 object tests, moving subjects to a testing pen or otherwise assuring that calves are alert at the

532 beginning of the test and not distracted by competing motivations will also help. For tests using

533 an unfamiliar human as the stimulus, moderate repeatability was only achieved for retreat

534 scores and not for likelihood of making contact with the person. None of the protocols assessed

535 provided consistently high repeatability, and results of neophobia tests seem to be dependent

536 on the specific stimuli chosen. For these reasons, we suggest that future research use multiple

537 tests to assess fearfulness or anxiety, using different stimuli.

539 Acknowledgements

We are grateful to the students and staff of the UBC Dairy Education and Research

542 Centre, especially to Alan Makarewicz, Tatiane Vito Camiloti, Nancy Chen, Justine Gallo, Annett

543 Gefrom, Clémence Messant, Pauline Gautier, Sara McNamara, Yasmine Yavari \& Ty Chapman

544 for help with calf care, and to João Cardoso Costa for helpful discussions. Funding was

545 provided by a Discovery Grant (RGPIN 262278-10) from the Natural Sciences and Engineering

546 Research Council of Canada (NSERC) to DMW.

$548 \quad$ References

550 Archer, J., 1973. Tests for emotionality in rats and mice: A review. Anim. Behav. 21, 205-235.

551 Austin, N.P., Rogers, L.J., 2007. Asymmetry of flight and escape turning responses in horses.

552 Laterality $12,464-474$. 
553 Barnett, J., Hemsworth, P., Newman, E., 1992. Fear of Humans and its Relationships with

554 Productivity in Laying Hens at Commercial Farms. Br. Poult. Sci. 33, 699-710.

555 Battini, M., Andreoli, E., Barbieri, S., Mattiello, S., 2011. Long-term stability of Avoidance

556 Distance tests for on-farm assessment of dairy cow relationship to humans in alpine traditional

557 husbandry systems. Appl. Anim. Behav. Sci. 135, 267-270.

558 Bell, A.M., Hankison, S.J., Laskowski, K.L., 2009. The repeatability of behaviour: a meta559 analysis. Anim. Behav. 77, 771-783.

560 Boivin, X., Le Neindre, P., Chupin, J.M., Garel, J.P., Trillat, G., 1992. Influence of breed and 561 early management on ease of handling and open-field behaviour of cattle. Appl. Anim. Behav.

562 Sci. 32, 313-323.

563 Bokkers, E.A.M., Leruste, H., Heutinck, L.F.M., Wolthuis-Fillerup, M., van der Werf, J.T.N.,

564 Lensink, B.J., van Reenen, C.G., 2009. Inter-observer and test-retest reliability of on-farm

565 behavioural observations in veal calves. Anim. Welf. 18, 381-390.

566 Bruton, A., Conway, J.H., Holgate, S.T., 2000. Reliability: What is it, and how is it measured?

567 Physiotherapy 86, 94-99.

568 Carlstead, K., Mellen, J., Kleiman, D.G., 1999. Black rhinoceros (Diceros bicornis) in US zoos: I.

569 Individual behavior profiles and their relationship to breeding success. Zoo Biol. 18, 17-34.

570 Carobrez, A.P., Bertoglio, L.J., 2005. Ethological and temporal analyses of anxiety-like

571 behavior: The elevated plus-maze model 20 years on. Neuroscience \& Biobehavioral Reviews;

572 Defensive Behavior 29, 1193-1205.

573 Carter, A.J., Feeney, W.E., Marshall, H.H., Cowlishaw, G., Heinsohn, R., 2013. Animal

574 personality: what are behavioural ecologists measuring? Biological Reviews 88, 465-475.

575 Cramer, M.C., Stanton, A.L., 2015. Associations between health status and the probability of 576 approaching a novel object or stationary human in preweaned group-housed dairy calves. J.

577 Dairy Sci. 98, 7298-7308. 
578 Credé, M., Harms, P., Niehorster, S., Gaye-Valentine, A., 2012. An evaluation of the

579 consequences of using short measures of the Big Five personality traits. J. Pers. Soc. Psychol.

$580 \quad 102,874-888$.

581 Dawson, G.R., Tricklebank, M.D., 1995. Use of the elevated plus maze in the search for novel

582 anxiolytic agents. Trends Pharmacol. Sci. 16, 33-36.

583 de Passillé, A.M., Rushen, J., 2005. Can we measure human-animal interactions in on-farm

584 animal welfare assessment? Appl. Anim. Behav. Sci. 92, 193-209.

585 De Rosa, G., Tripaldi, C., Napolitano, F., Saltalamacchia, F., Grasso, F., Bisegna, V., Bordi, A., 586 2003. Repeatability of some animal-related variables in dairy cows and buffaloes. Anim. Welfare $587 \quad 12,625-629$.

588 Duve, L.R., Weary, D.M., Halekoh, U., Jensen, M.B., 2012. The effects of social contact and 589 milk allowance on responses to handling, play, and social behavior in young dairy calves. J. 590 Dairy Sci. 95.

591 Ennaceur, A., 2014. Tests of unconditioned anxiety - Pitfalls and disappointments. Physiol. 592 Behav. 135, 55-71.

593 Farm Animal Welfare Council, 2009. Farm animal welfare in Great Britain: Past, present and 594 future.

595 Fina, M., Casellas, J., Manteca, X., Piedrafita, J., 2006. Analysis of temperament development 596 during the fattening period in the semi-feral bovine calves of the Alberes Massif. Animal

597 Research 55, 389-395.

598 Fonio, E., Benjamini, Y., Golani, I., 2012. Short and Long Term Measures of Anxiety Exhibit 599 Opposite Results. Plos One 7, e48414.

600 Forkman, B., Boissy, A., Meunier-Salauen, M.C., Canali, E., Jones, R.B., 2007. A critical review 601 of fear tests used on cattle, pigs, sheep, poultry and horses. Physiol. Behav. 92, 340-374.

602 Forkman, B., Keeling, L.J., 2009. Assessment of animal welfare measures for dairy cattle, beef 603 bulls and veal calves. Welfare Quality Reports 11, 1-314. 
604 Franks, B., Higgins, E.T., Champagne, F.A., 2012. Evidence for Individual Differences in

605 Regulatory Focus in Rats, Rattus norvegicus. J. Comp. Psychol. 126, 347-354.

606 Garner, J.P., 2005. Stereotypies and Other Abnormal Repetitive Behaviors: Potential Impact on 607 Validity, Reliability, and Replicability of Scientific Outcomes. ILAR 46, 106-117.

608 Gibbons, J., Lawrence, A., Haskell, M., 2009. Responsiveness of dairy cows to human 609 approach and novel stimuli. Appl. Anim. Behav. Sci. 116, 163-173.

610 Gnambs, T., 2014. A meta-analysis of dependability coefficients (test-retest reliabilities) for 611 measures of the Big Five. Journal of Research in Personality 52, 20-28.

612 Goodwin, L.D., Leech, N.L., 2006. Understanding correlation: Factors that affect the size of $r$.

613 The Journal of Experimental Education 74, 251-266.

614 Graunke, K.L., Langbein, J., Repsilber, D., Schoen, P., 2013. Objectively measuring behaviour

615 traits in an automated restraint-test for ungulates: towards making temperament measurable. J. 616 Agric. Sci. 151, 141-149.

617 Gray, J.A., 1987. The Psychology of Fear and Stress. Cambridge University Press, Cambridge. 618 Hall, C.S., 1936. Emotional behaviour in the rat. III. The relationship between emotionality and 619 ambulatory activity. J. Comp. Psych. 22, 345-352.

620 Haskell, M.J., Bell, D.J., Gibbons, J.M., 2012. Is the response to humans consistent over 621 productive life in dairy cows? Anim. Welfare 21, 319-324.

622 Hegelund, L., Sorensen, J.T., 2007. Measuring fearfulness of hens in commercial organic egg 623 production. Anim. Welfare 16, 169-171.

624 Hemsworth, P.H., Barnett, J.L., Coleman, G.J., Hansen, C., 1989. A Study of the Relationships 625 between the Attitudinal and Behavioral Profiles of Stockpersons and the Level of Fear of 626 Humans and Reproductive-Performance of Commercial Pigs. Appl. Anim. Behav. Sci. 23, 301627314

628 Hemsworth, P., Coleman, G., Barnett, J., Borg, S., 2000. Relationships between human-animal 629 interactions and productivity of commercial dairy cows. J. Anim. Sci. 78, 2821-2831. 
630 Jones, B., Boissy, A., 2011. Fear and other negative emotions, in: Appleby, M.C., Mench, J.A.,

631 Olsson, I.A.S., Hughes, B.O. (Eds.). CABI, Cambridge, UK, pp. 78-97.

632 Kilgour, R.J., Melville, G.J., Greenwood, P.L., 2006. Individual differences in the reaction of beef

633 cattle to situations involving social isolation, close proximity of humans, restraint and novelty.

634 Appl. Anim. Behav. Sci. 99, 21-40.

635 Lensink, B., Boivin, X., Pradel, P., Le Neindre, P., Veissier, I., 2000. Reducing veal calves'

636 reactivity to people by providing additional human contact. J. Anim. Sci. 78, 1213-1218.

637 Lister, R.G., 1987. The use of a Plus-Maze to Measure Anxiety in the Mouse.

638 Psychopharmacology (Berl. ) 92, 180-185.

639 MacKay, J.R.D., Haskell, M.J., Deag, J.M., van Reenen, K., 2014. Fear responses to novelty in 640 testing environments are related to day-to-day activity in the home environment in dairy cattle.

641 Appl. Anim. Behav. Sci. 152, 7-16.

642 Martin, P., Bateson, P., 2007. Measuring Behaviour: An Introductory Guide. Cambridge

643 University Press, Cambridge, UK.

644 Mazurek, M., McGee, M., Crowe, M.A., Prendiville, D.J., Boivin, X., Earley, B., 2011.

645 Consistency and stability of behavioural fear responses of heifers to different fear-eliciting

646 situations involving humans. Appl. Anim. Behav. Sci. 131, 21-28.

647 McGuirk, S., 2013. Calf health scoring chart. 2015.

648 Meagher, R.K., 2009. Observer ratings: Validity and value as a tool for animal welfare research.

649 Appl. Anim. Behav. Sci. 119, 1-14.

650 Meagher, R.K., Daros, R.R., Costa, J.H.C., von Keyserlingk, Marina A. G., Hötzel, M.J., Weary,

651 D.M., 2015. Effects of Degree and Timing of Social Housing on Reversal Learning and

652 Response to Novel Objects in Dairy Calves. PLoS ONE 10, e0132828.

653 Meagher, R.K., Duncan, I., Bechard, A., Mason, G.J., 2011. Who's afraid of the big bad glove?

654 Testing for fear and its correlates in mink. Appl. Anim. Behav. Sci. 133, 254-264. 
655 Millman, S.T., 2007. Sickness behaviour and its relevance to animal welfare assessment at the 656 group level. Anim. Welfare 16, 123-125.

657 Misslin, R., Ropartz, P., 1981. Responses in Mice to a Novel Object. Behaviour 78, 169-177.

658 Mroczek, D.K., 2007. The analysis of longitudinal data in personality research, in: Robins, R.W.,

659 Fraley, R.C., Krueger, R.F. (Eds.). Guilford Press, New York, USA, pp. 543-556.

660 Ohl, F., 2003. Testing for anxiety. Clinical Neuroscience Research 3, 233-238.

661 Ramos, A., 2008. Animal models of anxiety: do I need multiple tests? Trends Pharmacol. Sci. $662 \quad 29,493-498$.

663 Roberts, B., DelVecchio, W., 2000. The rank-order consistency of personality traits from 664 childhood to old age: A quantitative review of longitudinal studies. Psychol. Bull. 126, 3-25.

665 Robins, A., Phillips, C., 2010. Lateralised visual processing in domestic cattle herds responding 666 to novel and familiar stimuli. Laterality $15,514-534$.

667 Rodgers, R.J., 1997. Animal models of 'anxiety': where next? Behav. Pharmacol. 8, 477-496.

668 Rousing, T., Ibsen, B., Sorensen, J.T., 2005. A note on: On-farm testing of the behavioural 669 response of group-housed calves towards humans; test-retest and inter-observer reliability and 670 effect of familiarity of test person. Appl. Anim. Behav. Sci. 94, 237-243.

671 Russell, P.A., 1973. Relationships between exploratory behaviour and fear: a review. British 672 Journal of Psychology 63, 417-433.

673 Schönbrodt, F.D., Perugini, M., 2013. At what sample size do correlations stabilize? J. Res.

674 Personality 47, 609-612.

675 Schuetz, K.E., Hawke, M., Waas, J.R., McLeay, L.M., Bokkers, E.A.M., van Reenen, C.G.,

676 Webster, J.R., Stewart, M., 2012. Effects of human handling during early rearing on the

677 behaviour of dairy calves. Anim. Welfare 21, 19-26.

678 Scott, E.M., Nolan, A.M., Fitzpatrick, J.L., 2001. Conceptual and Methodological Issues Related

679 to Welfare Assessment: A Framework for Measurement. Acta Agriculturae Scandinavica,

680 Section A - Animal Science 51, 5-10. 
681 Smith, B.R., Blumstein, D.T., 2008. Fitness consequences of personality: a meta-analysis.

682 Behav. Ecol. 19, 448-455.

683 Sousa, N., Almeida, O., Wotjak, C., 2006. A hitchhiker's guide to behavioral analysis in 684 laboratory rodents. Genes Brain and Behavior 5, 5-24.

685 Swiergiel, A.H., Dunn, A.J., 2007. Effects of interleukin-1 $\beta$ and lipopolysaccharide on behavior 686 of mice in the elevated plus-maze and open field tests. Pharmacology Biochemistry and 687 Behavior 86, 651-659.

688 Sylvers, P., Lilienfeld, S.O., LaPrairie, J.L., 2011. Differences between trait fear and trait 689 anxiety: Implications for psychopathology. Clin. Psychol. Rev. 31, 122-137.

690 Taylor, J., 1987. Kendall and Spearman Correlation-Coefficients in the Presence of a Blocking 691 Variable. Biometrics 43, 409-416.

692 Tecott, L.H., Nestler, E.J., 2004. Neurobehavioral assessment in the information age. Nat.

693 Neurosci. 7, 462-466.

694 Turner, S.P., Navajas, E.A., Hyslop, J.J., Ross, D.W., Richardson, R.I., Prieto, N., Bell, M., 695 Jack, M.C., Roehe, R., 2011. Associations between response to handling and growth and meat 696 quality in frequently handled Bos taurus beef cattle. J. Anim. Sci. 89, 4239-4248.

697 Van Reenen, C.G., Van der Werf, J.T.N., O'Connell, N.E., Heutinck, L.F.M., Spoolder, H.A.M., 698 Jones, R.B., Koolhaas, J.M., Blokhuis, H.J., 2013. Behavioural and physiological responses of 699 heifer calves to acute stressors: Long-term consistency and relationship with adult reactivity to 700 milking. Appl. Anim. Behav. Sci. 147, 55-68.

701 Van Reenen, C.G., Hopster, H., Van der Werf, J.T.N., Engel, B., Buist, W.G., Jones, R.B.,

702 Blokhuis, H.J., Korte, S.M., 2009. The benzodiazepine brotizolam reduces fear in calves

703 exposed to a novel object test. Physiol. Behav. 96, 307-314.

704 Van Reenen, C.G., O'Connell, N.E., Van der Werf, J.T.N., Korte, S.M., Hopster, H., Jones, R.B., 705 Blokhuis, H.J., 2005. Responses of calves to acute stress: Individual consistency and relations 706 between behavioral and physiological measures. Physiol. Behav. 85, 557-570. 
707 Van Reenen, C., Engel, B., Ruis-Heutinck, L., Van der Werf, J., Buist, W., Jones, R., Blokhuis,

708 H., 2004. Behavioural reactivity of heifer calves in potentially alarming test situations: a

709 multivariate and correlational analysis. Appl. Anim. Behav. Sci. 85, 11-30.

710 Waiblinger, S., Boivin, X., Pedersen, V., Tosi, M., Janczak, A.M., Visser, E.K., Jones, R.B.,

711 2006. Assessing the human-animal relationship in farmed species: A critical review. Appl. Anim.

712 Behav. Sci. 101, 185-242.

713 Wemelsfelder, F., Lawrence, A.B., 2001. Qualitative Assessment of Animal Behaviour as an

714 On-Farm Welfare-monitoring Tool. Acta Agriculturae Scandinavica, Section A - Animal

715 Science $51,21-25$.

716 Whay, H.R., Main, D.C.J., Green, L.E., Webster, A.J.F., 2003. Animal-based measures for the

717 assessment of welfare state of dairy cattle, pigs and laying hens: consensus of expert opinion.

718 Anim. Welfare 12, 205-217.

719 Winckler, C., Brinkmann, J., Glatz, J., 2007. Long-term consistency of selected animal-related

720 welfare parameters in dairy farms. Anim. Welfare 16, 197-199.

721 Winckler, C., Capdeville, J., Gebresenbet, G., Horning, B., Roiha, U., Tosi, M., Waiblinger, S.,

722 2003. Selection of parameters for on-farm welfare-assessment protocols in cattle and buffalo.

723 Anim. Welfare 12, 619-624.

724 Windschnurer, I., Barth, K., Waiblinger, S., 2009. Can stroking during milking decrease

725 avoidance distances of cows towards humans? Anim. Welfare 18, 507-513.

726 Windschnurer, I., Boivin, X., Waiblinger, S., 2009. Reliability of an avoidance distance test for

727 the assessment of animals' responsiveness to humans and a preliminary investigation of its

728 association with farmers' attitudes on bull fattening farms. Appl. Anim. Behav. Sci. 117, 117-

729127.

730 Windschnurer, I., Schmied, C., Boivin, X., Waiblinger, S., 2008. Reliability and inter-test

731 relationship of tests for on-farm assessment of dairy cows' relationship to humans. Appl. Anim.

732 Behav. Sci. 114, 37-53. 
733 Wurbel, 2002. Behavioral phenotyping enhanced - beyond (environmental) standardization.

734 Genes, brain, and behavior 1, 3.

736 Tables

\section{Table 1}

740 Fear scoring system in Experiment 3 based on stage at which the calf retreated from the

741 approaching experimenter. The experimenter approached the calf or calves in the home pen, in

742 a standardized way each time, and the calf was given a total of 2 min to approach or retreat.

\begin{tabular}{ll}
\hline Score & Description \\
\hline 9 & Retreat before arrive at pen \\
8 & Retreat when face pen \\
7 & Retreat when speak \\
$4-6$ & Retreat during approach (each step the experimenter took towards the pen before a \\
& retreat reducing the score by 1) \\
3 & Retreat when reached front of pen \\
2 & Retreat when extend arm \\
1 & Retreat during remainder of test \\
0 & No retreat \\
\hline
\end{tabular}




\section{Table 2}

748 Spearman correlation coefficients from Experiment 3. Coefficients describe the repeatability of

749 responses to the approaching human test on commercial farms, depending on the response

750 variable and exclusion criteria. Each of 14 farms was tested on two occasions. The highest

751 coefficient for each response variable is indicated in bold.

752

\begin{tabular}{|c|c|c|c|c|}
\hline \multirow[b]{2}{*}{ Response variable } & \multicolumn{4}{|c|}{ Exclusion criteria } \\
\hline & $\begin{array}{l}\text { Sick } \\
\text { calves } \\
\text { included }\end{array}$ & $\begin{array}{l}\text { Calves } \\
\text { with } \\
\text { diarrhoea } \\
\text { excluded }\end{array}$ & $\begin{array}{l}\text { Calves } \\
\text { with } \\
\text { coughs } \\
\text { excluded }\end{array}$ & $\begin{array}{l}\text { Both } \\
\text { excluded }\end{array}$ \\
\hline $\begin{array}{l}\text { Proportion of calves that } \\
\text { made contact }\end{array}$ & 0.222 & 0.279 & 0.332 & 0.253 \\
\hline $\begin{array}{l}\text { Proportion retreating when } \\
\text { arm extended or before } \\
\text { Proportion retreating before }\end{array}$ & 0.508 & 0.450 & 0.494 & 0.486 \\
\hline arm extended & 0.516 & 0.477 & 0.521 & 0.486 \\
\hline Average retreat score & 0.538 & 0.587 & 0.582 & 0.560 \\
\hline
\end{tabular}




\section{$755 \quad$ Figures}

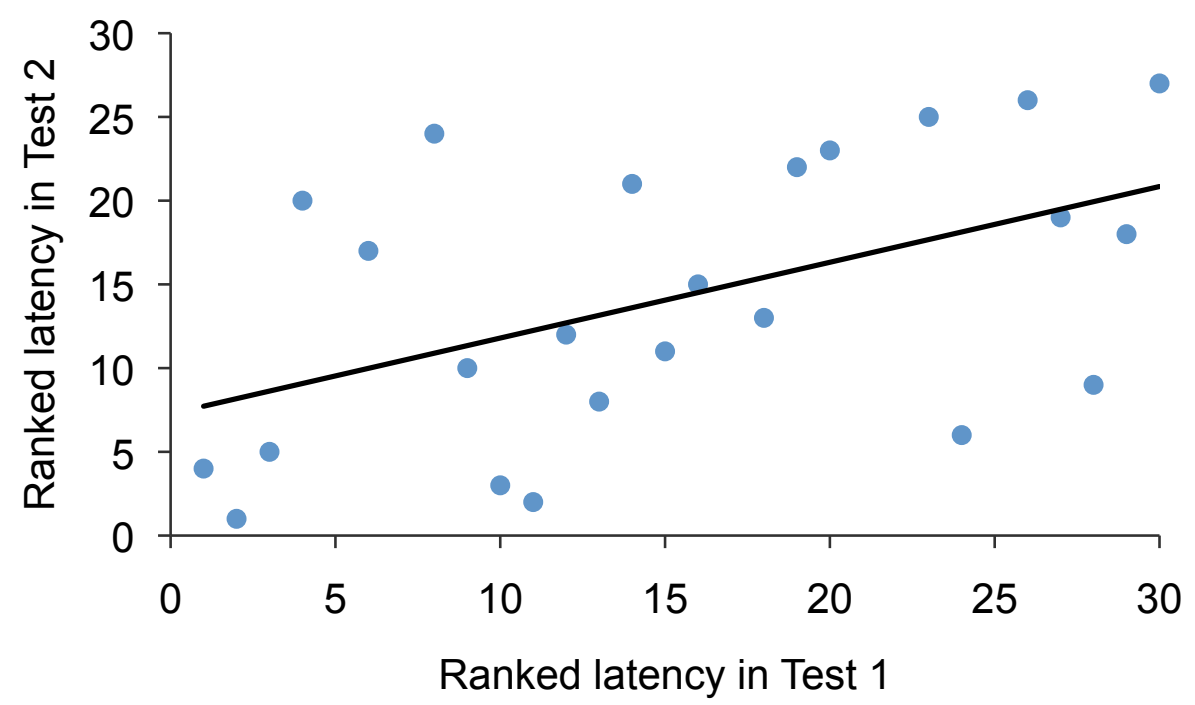

756

757 Fig. 1. Rank correlation between dairy calves' latencies to touch a novel object across two tests

758 in Experiment 1. Calves were tested at approximately 41 and $62 \mathrm{~d}$ of age, with the object being 759 a colourful ball. 

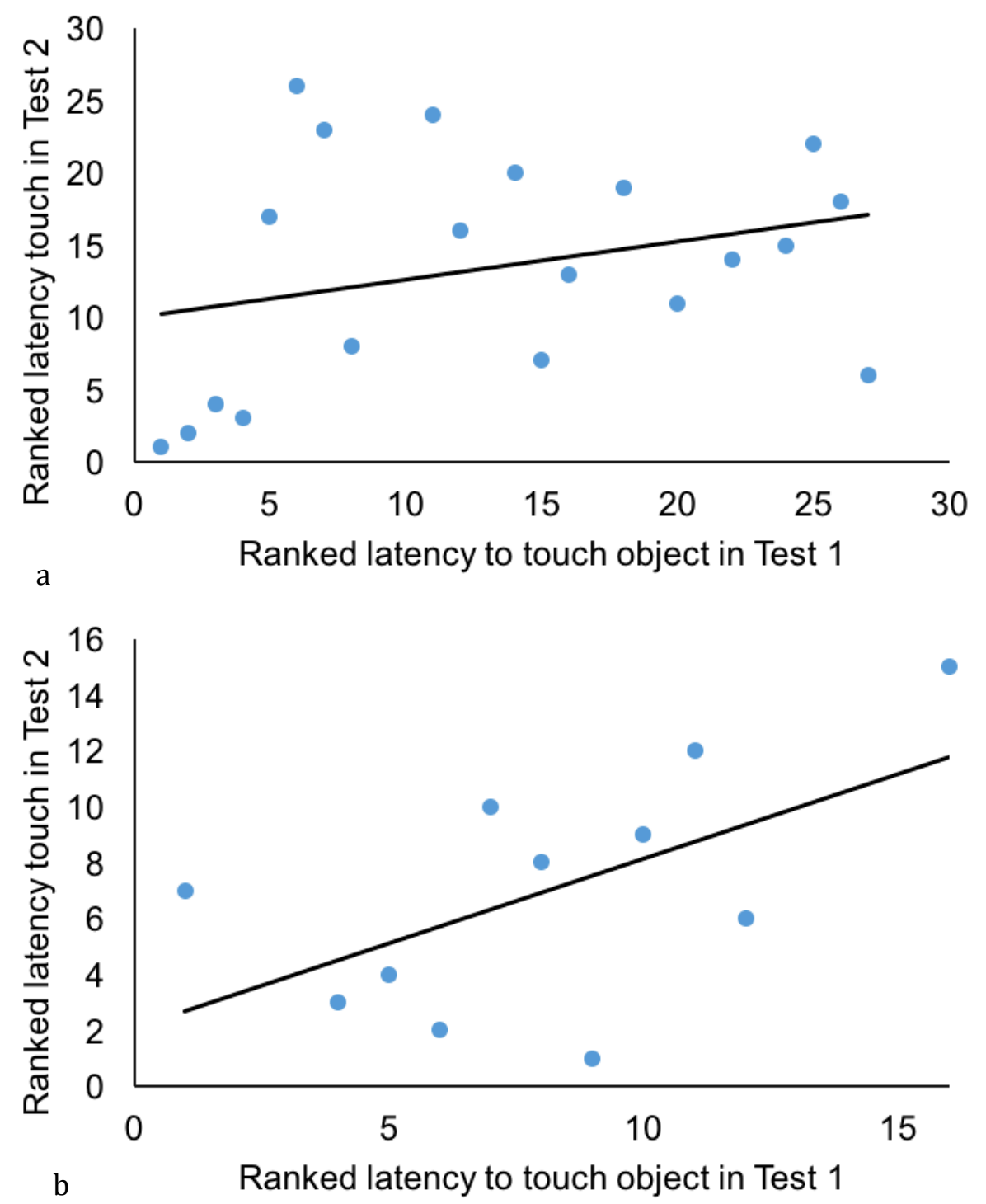

762 Fig. 2. Rank correlation between calves' latencies to touch a novel object across two tests in

763 Experiments $2 a$ and b. Calves were tested at approximately 36 and $43 \mathrm{~d}$ of age, using a

764 different object each time in a) versus the same object in b). 


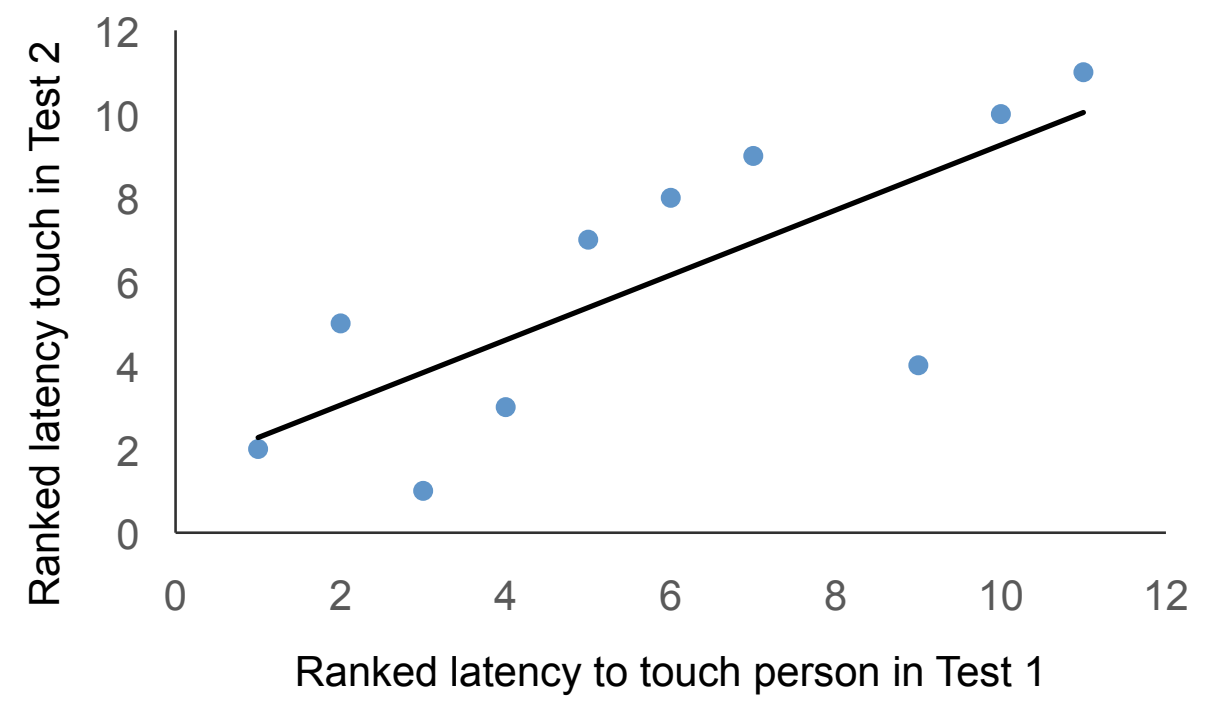

766

767 Fig. 3. Rank correlation between calves' latencies to touch a familiar handler across two tests in

768 Experiment 2b. Calves were let out of their pens and given up to $3 \mathrm{~min}$ to touch a stationary

769 person.

770 